\title{
Non-alkolik nedenlere bağlı gelişen Wernicke ensefalopatisinde manyetik rezonans görüntüleme bulguları
}

Magnetic resonance imaging findings of Wernicke's encephalopathy in non-alcoholic patients

\author{
Cenk Eraslan ${ }^{1} \quad$ Ayşe Güler ${ }^{2}\left(\mathbb{D} \quad\right.$ Erman Kurt $^{3} \mathbb{D} \quad$ Cem Çallı $^{1}$ Ömer Kitiş ${ }^{1}$ \\ ${ }^{1}$ Ege Üniversitesi Tıp Fakültesi, Radyodiagnostik Anabilim Dalı, İzmir, Türkiye \\ ${ }^{2}$ Ege Üniversitesi Tıp Fakültesi, Nöroloji Anabilim Dalı, İzmir, Türkiye \\ ${ }^{3}$ Menemen Devlet Hastanesi, Radyoloji Kliniği, İzmir, Türkiye
}

\section{Öz}

Amaç: Wernicke ensefalopatisi (WE) tiamin (vitamin B1) eksikliğine bağlı olarak gelişen nöropsikiyatrik bir hastalıktır. Sıklıkla kronik alkolizme bağlı olarak görülmekle birlikte tiamin alım veya emilim eksikliğine neden olan her türlü durumda ortaya çıkabilir. Bu çalışmanın amacı tedavi edilmediği takdirde ölüme kadar giden ciddi sonuçlar doğurabilen bu hastalığın tanı ve izleminde manyetik rezonans görüntülemenin (MRG) önemini vurgulamaktır.

Gereç ve Yöntem: Çalışmamıza kurumumuzda hospitalize edilen ve non-alkolik nedenlerle WE tanısı alan 10 hasta dahil edildi. Hastaların MRG tetkiklerinde tutulum yerlerinin lokalizasyonuna göre tipik ve atipik bulguların varlığı değerlendirildi. Ayrıca eşlik eden difüzyon kısıtılığı varlığı araştıııldı.

Bulgular: Kraniyal MRG'de tipik tutulum alanları 10 hastada bilateral talamus mediali, 8 hastada bilateral mamiller cisim, 7 hastada periakuaduktal alan/tektal plak olarak tespit edildi. Atipik tutulum alanları ise 5 hastada forniks, 4 hastada serebral korteks, 2 hastada putamen, 1 hastada ise serebellum tutulumu şeklinde idi. 8 hastada bu bulgulara difüzyon kısıtıı̆ğı eşlik etmekteydi.

Sonuç: Sunulan çalışmanın sonuçlarına göre, non-alkolik WE tanılı olgularda MRG'de, alkolik WE tanılı olgularla benzer bulgular gözlenmektedir. Bu hastaların tanı ve tedavilerinin gecikmemesi amacıyla MR görüntülemenin kullanımının önemli rol oynadığı sonucuna varılmıştır.

Anahtar Sözcükler: Wernicke ensefalopatisi, manyetik rezonans görüntüleme, tiamin eksikliği.

\section{Abstract}

Aim: Wernicke encephalopathy (WE) is a neuropsychiatric disease that develops due to the deficiency of thiamine (vitamin B1). It is often observed due to chronic alcoholism; however it can arise in any condition that causes deficiency in the intake or absorption of thiamine. The aim of this study is to emphasize the importance of magnetic resonance imaging (MRI) in the diagnosis and follow-up of this disease which can even lead to death when it is not treated.

Materials and Methods: Ten patients, who had been hospitalized in our institution and diagnosed as WE due to non-alcoholic reasons, were included in our study. In the MRI examinations of the patients, typical and atypical findings were evaluated according to the localization of involvement areas. The existence of accompanying diffusion restriction was evaluated.

Results: Typical involvement areas in cranial MRI were determined as bilateral medial thalamus in 10 patients, bilateral mammillary bodies in 8 patients, periaqueductal area/tectal plate in 7 patients. Atypical involvement areas were determined as fornix in 5 patients, cerebral cortex in 4 patients, putamen in 2 patients and cerebellum involvement in 1 patient. These findings were accompanied by diffusion restriction in 8 patients.

Conclusion: According to the results of the presented study, cranial MRI has similar findings in alcoholic and nonalcoholic WE patients. We conclude that, the utilization of cranial MRI in these patients has an important role to prevent the latency of the diagnosis and treatment.

Keywords: Wernicke encephalopathy, magnetic resonance imaging, thiamine deficiency.

\footnotetext{
Yazışma Adresi: Cenk Eraslan

Ege Üniversitesi Tıp Fakültesi, Radyodiagnostik Anabilim Dalı,

İzmir, Türkiye

E-mail: eraslancenk@hotmail.com

Makalenin Geliş Tarihi: 13.03.2018 Kabul Tarihi: 09.04.2018
} 


\section{Giriş}

Wernicke ensefalopatisi (WE) tiamin (vitamin B1) eksikliğine bağlı olarak gelişen nöropsikiyatrik bir hastalıktır. Sıklıkla kronik alkolizme bağlı olarak görülmekle birlikte tiamin alım veya emilim eksikliğine neden olan her türlü durumda ortaya çıkabilir. Uzamış kusma ve açlık, gastrointestinal cerrahiler, uzun süreli parenteral beslenme ve bu sırada multivitamin desteğinin yetersiz yapılması non-alkolik grubun nedenleri arasında sayılabilir (1-5). Ani gelişen bilinç değişiklikleri, nöro-oftalmoplejik bulgular ve ataksi ile karakterize klasik bir triada sahip olsa da, bu triad olguların küçük bir bölümünde bulunmaktadır $(6,7)$. Yapılan çalışmalar olguların sadece \%20'sinin yaşamları sırasında tanı aldığını ve büyük çoğunluğunun postmortem tanındığını göstermektedir (8). Hastalığın prognozu erken dönemde intravenöz tiamin replasmanına bağlı olarak değişmektedir. Manyetik rezonans görüntülemede (MRG) karakteristik olarak T2 ve Fluid Attenuated Inversion Recovery (FLAIR) ağırıklı görüntülerde sıklıkla talamus mediali, mamiller cisim, periakuaduktal alan ve tektal plakta simetrik sinyal artışları izlenir. Difüzyon ağırlıklı görüntülemede tutulum izlenen bu alanlarda difüzyon kısıtlanması görünüme eşlik edebilir. Bu nedenle kraniyal MRG tanıyı doğrulamada önemli bir rol oynamaktadır (7).

$\mathrm{Bu}$ çalışmanın amacı tedavi edilmediği takdirde ölüme kadar giden ciddi sonuçlar doğurabilen bu hastalığın tanı ve izleminde MRG'nin önemini vurgulamaktır.

\section{Gereç ve Yöntem}

Çalışmamıza 2010 ile 2017 tarihleri arasında Ege Üniversitesi Tıp Fakültesi'nde hospitalize edilen ve nonalkolik nedenlerle WE tanısı alan 5'i erkek, 5'i kadın 10 hasta dahil edildi. Veri tabanına kaydedilmiş olan hasta bilgileri retrospektif olarak incelendi. Hastaların yaşı, cinsiyeti, etiyolojik faktörleri, klinik bulguları kaydedildi. Radyoloji ünitemizde hastalara 1,5 tesla MRG cihazı (Symphony Vision, Siemens, Erlangen, Germany) ve 3 tesla MRG (Verio, Siemens, Erlangen, Germany) ile T1, T2 ve FLAIR ağırlıklı sekanslar ile yapılan konvansiyonel kraniyal MR inceleme ile difüzyon ağırlıklı seriler retrospektif olarak değerlendirildi. Bu değerlendirme iki ayrı radyoloji uzmanı tarafından gerçekleştirildi. MRG bulguları, tutulum yerlerinin lokalizasyonuna göre; tipik (talamus, mamiller cisim ve periakuaduktal alan/tektum) ve atipik (serebellum, forniks, putamen, serebral korteks) tutulum şeklinde kaydedildi. Ayrıca tutulum yerlerinin herhangi birinde eşlik eden difüzyon kısıtılığı varlığı değerlendirildi. Hastaların tiamin replasmanı sonrası klinik takip ve laboratuvar bulguları kayıt altına alındı. Var ise takip görüntüleme bulguları incelenerek bulgular değerlendirildi.

\section{Bulgular}

Çalışmamız WE tanısı alan 5'i erkek (\%50), 5’i kadın (\%50) toplam 10 hasta ile gerçekleştirildi. Hastaların tanı aldıkları andaki yaşları 15 ile 65 arasında değişmekte olup, ortalama yaş 49.5 idi.

Hastaların nörolojik muayenesinde; 9 hastada bilinç bulanıklığı (değişik derecelerde), 5 hastada oftalmopleji bulguları (nistagmus), 3 hastada ataksi, 3 hastada vertigo, 1 hastada dizartri, 1 hastada ise işitme kaybı vardı. Tipik klinik triad 2 olguda tespit edildi.

Kraniyal MRG'de tipik tutulum alanları 10 hastada bilateral talamus mediali, 9 hastada bilateral mamiller cisim, 7 hastada periakuaduktal alan/tektal plak olarak tespit edildi. Atipik tutulum alanları ise 5 hastada forniks, 4 hastada serebral korteks, 2 hastada putamen, 1 hastada ise serebellum tutulumu şeklinde idi. 8 hastada ise bu bulgulara tutulum saptanan alanlarda izlenen difüzyon kısıtıılı̆ı eşlik etmekteydi (Tablo-1).(Şekil-1).

Tüm bulgular ışığında tiamin replasmanı yapılan olguların; 3'ünde tedaviye tama yakın yanıt izlenirken, 3 hastada ise kısmi yanıt gözlendi. Dört hasta primer hastalıkları ve komorbiditeler nedeniyle kaybedildi. (Şekil-2).

Tablo-1. Hastaların Kraniyal Manyetik Rezonans Görüntüleme Bulguları.

\begin{tabular}{|c|c|c|c|c|c|c|c|c|}
\hline & \multicolumn{3}{|c|}{ T2 - Tipik bulgular } & \multicolumn{4}{|c|}{ T2 - Atipik bulgular } & \multirow{2}{*}{$\begin{array}{l}\text { Difüzyon ağırlıkl } \\
\text { görüntüleme }\end{array}$} \\
\hline & Talamik & $\begin{array}{l}\text { Mamiller } \\
\text { cisim }\end{array}$ & $\begin{array}{l}\text { Periakuadukt / } \\
\text { tektal plak }\end{array}$ & $\begin{array}{l}\text { Serebral } \\
\text { korteks }\end{array}$ & Serebellum & $\begin{array}{c}\text { Bazal } \\
\text { ganglion }\end{array}$ & Forniks & \\
\hline Olgu - 1 & + & + & - & - & - & - & - & + \\
\hline Olgu - 2 & + & + & + & + & - & - & + & + \\
\hline Olgu - 3 & + & + & - & - & - & - & - & - \\
\hline Olgu - 4 & + & + & - & + & - & - & - & + \\
\hline Olgu - 5 & + & + & + & + & - & - & - & + \\
\hline Olgu - 6 & + & + & + & - & - & - & + & + \\
\hline Olgu - 7 & + & + & + & - & - & - & + & - \\
\hline Olgu - 8 & + & + & + & - & - & - & - & + \\
\hline Olgu - 9 & + & - & + & + & + & + & + & + \\
\hline Olgu - 10 & + & + & + & - & - & + & + & + \\
\hline
\end{tabular}




\section{Tartışma}

WE, tiamin eksikliğine bağlı ortaya çıkan, tanı ve tedavisindeki gecikme sonucunda kalıcı ağır nörolojik hasar oluşturan ve ölüm ile sonuçlanabilen nöropsikiyatrik bir bozukluktur (6).

Tiamin (vitamin B1) suda çözünen, başta karaciğer olmak üzere vücutta depo edilebilen esansiyel bir vitamindir. Membran bütünlüğü ve membranlar arası osmotik gradientin korunmasında rol oynamaktadır (9). Tiamin, krebs ve pentoz fosfat döngüsünde yer alan 3 anahtar enzim olan $\alpha$-ketoglutarat dehidrogenaz ve piruvat dehidrojenaz kompleksi ile transketolaz için önemli bir ko-faktördür (10). Sağlıklı bir yetişkinin tiamin intiyacı günde yaklaşık 1-2 mg'dır. Vücudun tiamin rezervleri sadece 30-50 mg olduğundan, 3-4 haftadan uzun süren beslenme bozuklukları vitamin depolarını tamamen tüketebilir (11). WE en sık kronik alkolizm ile birlikte görülmektedir. Aslında kronik alkol kullanımı doğrudan tiamin eksikliğinden sorumlu değildir. Sıklıkla bu duruma eşlik eden yetersiz beslenme, mukozal düzeyde düşük tiamin emilim hızı, karaciğer fonksiyon bozuklukları ve artmış tiamin metabolizması, vitamin eksikliği gelişimine neden olabilir (11). Bununla birlikte, alkol ile ilişkili olmayan birçok klinik durumda da tiamin eksikliği gözlenebilir (Tablo-2). Olgularımızın etiyolojisinde; primer hastalıkları zemininde gelişen 5 hastada parenteral beslenme, 4 hastada yetersiz beslenme, 1 hastada ise tekrarlayan kusma yer almakta idi (Tablo-3).

Tablo-2. Non-Alkolik Wernicke Ensefalopatisi Nedenleri.

Gastrointestinal cerrahiler
Obezite cerrahisi
Gastrik bypass cerrahisi
Gastrojejunostomi
Uzun süreli Açlık
Sosyoekonomik nedenler
Uzun süreli diyet
Geriatrik hastalar
Uzun süreli kusma
Hiperemezis gravidarum
Kemoterapi
İyatrojenik
Total parenteral beslenme
Uzamış intravenöz beslenme
Aşırı glukoz yüklemeleri
Kronik Hemodiyaliz
Pankreatit
Psikiyatrik bozukluklar
Anoreksiya nervoza
Şizofreni

Tablo-3. Hastaların Primer Klinik Tanıları ve Wernicke Ensefalopatisi Gelişmesini Tetikleyen Nedenler.

\begin{tabular}{lll}
\hline & Primer hastalık & Wernicke ensefalopatisi etiyolojisi \\
\hline Olgu - 1 & Primeri bilinmeyen nöroendokrin karaciğer tümörü - & Uzun süreli parenteral nutrisyon \\
Olgu - 2 & Iyatrojenik safra yolu yaralanması & Malnutrisyon \\
Olgu - 3 & Larinks malign neoplazmı (opere) & Malnutrisyon \\
Olgu - 4 & Mide malign neoplazmı (opere) & Uzun süreli parenteral nutrisyon \\
Olgu - 5 & Koledok alt uç tümörü (opere) & Kusma \\
Olgu - 6 & SMV* trombozu - ileus & Uzun süreli parenteral nutrisyon \\
Olgu - 7 & Obezite cerrahisi sonrası anastamoz darlığı & Uzun süreli parenteral nutrisyon \\
Olgu - 8 & Bilateral hidronefroz sonrası nefrostomi & Malnutrisyon \\
Olgu - 9 & Akut kolanjit - Hepatit C & Uzun süreli parenteral nutrisyon \\
Olgu - 10 & Vaskülit - akut pankreatit & Malnutrisyon \\
\hline
\end{tabular}

*Superior mezenterik ven.

Patolojik açıdan değerlendirildiğinde akut WE'de, tiamin eksikliği ile osmotik gradient bozularak intraselüler ve ekstraselüler boşluklarda ödem oluşur. Ayrıca değişken derecelerde nekroz, demyelinizasyon, vasküler ve mikroglial proliferasyon, peteşiyal kanamalar ve bu değişiklikleri takiben kan-beyin bariyeri işlev bozukluğu meydana gelir. Zamanında tiamin desteği yapılmadığı takdirde hücre ölümleri gerçekleşir. Bu bulgular WE'nin patojenik mekanizmasına hem vazojenik hem de sitotoksik ödemin katkıda bulunduğunu göstermektedir $(12,13)$. Beyinde en belirgin patolojik değişiklikler, daha gevşek yapıda kan beyin bariyerine sahip ve tiamin ilişkili glukoz ve oksidatif metabolizmanın daha yüksek oranda olduğu bölgelerde izlenir (9). Bu bölgeler üçüncü ventrikül çevresinde yerleşim gösteren talamus mediali, mamiller cisim, periakuaduktal gri cevher ile tektal plaktır (7-13). Bu alanların tutulumu WE için karakteristik olarak tanımlanmaktadır (7)

WE için akut başlangıçlı klasik bir klinik triad tanımlanmıştır. Farklı derecelerde bilinç bulanıklığı, nöro-oftalmolojik semptomlar ve ataksiden oluşan bu triad olguların yalnızca yaklaşık \%16-38' inde mevcuttur 
(6-7). En sık eşlik eden klinik bulgu, mental durum değişiklikleridir. $\mathrm{Bu}$ değişiklikler hafif oryantasyon bozukluğundan komaya kadar geniş bir yelpazeyi içerir. Bizim olgularımızın 9'unda (\%90) literatür ile uyumlu olarak farklı derecelerde bilinç bozuklukları mevcut idi (14). Oftalmolojik bozukluklar arasında en sık görüleni ise nistagmustur. Görme keskinliğinde azalma, diplopi, oküler kas felçleri, retinal hemoraji, papil ödem, anizokori diğer bulgular arasında sayılabilir (15). Bizim çalışmamızda 5 hastada nistagmus tespit edilmiş olup, başka bir oftalmopleji bulgusuna rastlanmamıştır. Denge bozuklukları da hafiften şiddetliye değişik derecelerde gözlenebilir. WE'ye eşlik eden diğer klinik bulgular arasında karın ağrısı, polinöropati, dizartri, hipotermi, işitme kayıpları, vertigo, epileptik nöbetler sayılabilir.

WE klinik olarak tanısı konulan bir hastalıktır. Kırmız kan hücrelerinde transketolaz aktivitesi ve kan tiamin fosfat düzeyi ölçümü tanıya yardımcı parametrelerdir. Ancak bu ölçümler, teknik zorluklar ve düşük özgüllük nedeniyle sınırlı kullanıma sahiptir. Bu nedenle kraniyal MRG, WE tanısı şüpheli olgularda karakteristik görüntüleme bulgularıyla tanının desteklenmesinde kritik bir rol oynar. Yüksek kontrast rezolüsyonu sayesinde kraniyal MRG radyolojik görüntülemede altın standarttır. Kraniyal MRG bulguları patolojik değişiklikler ile uyumlu olarak tipik ve atipik bulgular şeklinde iki gruba ayrılır. Medial talamus, mamiller cisim, periakuaduktal gri cevher ile tektal plakta T2 ve FLAIR sekanslarında bilateral ve simetrik sinyal artışları tipik bulgu olarak kabul edilir. Serebellar hemisfer, serebellar vermis, dentat nukleus, kraniyal sinir nukleusları, korpus kallozum splenium kesiminde, forniks, serebral korteks, bilateral red nukleuslar ve putamen patolojik olarak daha az tutulan bölgeler olup bu bölgelerdeki sinyal değişiklikleri atipik gruba dahil edilir.

Tipik görüntüleme bulguları arasında en sık medial talamus (3. ventrikül komşuluğuna uyan bölümü) tutulumu yer alır. Bu bölgedeki sinyal değişiklikleri neredeyse her zaman hastalığın diğer tipik bulgularıyla birlikte gözlenir. Çalışmamızda literatür ile uyumlu olarak hastaların tamamında talamus medialinde patolojik sinyal değişikliği gözlendi (16). Ayrıca bu bölgenin tutulumu oldukça nadir de olsa WE'nin tek bulgusu olarak ortaya çıkabilir (7). Buna paralel olarak bizim olgularımızdan da birinde sadece bu bölgede sinyal değişikliği gözlendi. Zhong ve ark. (10) 2005 'te yayımlanan non-alkolik 6 hasta ile yaptıkları çalışmalarında, medial talamus ve kaudat nukleus (baş kısmı) tutulumunu bilinç değişikliklerinden koma ile ilişkilendirmiştir. Ancak tamamında talamus medialinde tutulum gözlenen olgularımızın altısında koma hali mevcut olmamasının yanı sıra bu olgularda tedavi sonrasında bilinç bulgularında kısmi veya tama yakın regresyon gözlendi. Diğer bir tipik bulgu olan mamiller cisim tutulumu ise bazı yayınlarda daha çok alkoliklerde bildirilmiştir (7). Buna karşın bizim olgularımızın tamamına yakınında (9/10) mamiller cisimde simetrik sinyal değişikliği gözlendi.

Atipik görüntüleme bulgularından serebellar tutulum; hem alkolik hem de non-alkolik hasta grubunda, tipik veya atipik sinyal değişiklikleriyle birlikte gözlenebilir. MR görüntülemede serebellum tutulumu oldukça nadirdir. Ancak patolojik çalışmalar, WE olgularının yarısından fazlasında serebellumun etkilendiğini göstermektedir (17). Kraniyal sinir tutulumu ile ilgili birkaç yayınlanmış olgu mevcut olup; tutulan sinirler 6., 7., 8. ve 12. kraniyal sinirlerdir $(7,18)$. Bu değişiklikler her zaman non-alkolik hastalarda ve hastalığın tipik diğer sinyal değişiklikleri ile beraber gözlenmiştir (7). Olgularımızın hiçbirisinde kraniyal sinir tutulumu mevcut değildi. Kortikal tutulum literatürde geri döndürülemeyen hasar ve kötü prognoza işaret eder $(10,16)$. Bununla birlikte Chui ve ark. (19) tekrar bilincini kazanan akut pankreatitli bir WE olgusunu bildirmiştir. Çalışmamızı gözden geçirdiğimizde, kortikal tutulum saptanan 4 olgumuzun 3 'ü WE tanısı konulmasını takiben kısa süre içerisinde kaybedildi. Koledok alt uç tümörü nedeniyle opere olan ve uzun süreli parenteral nutrisyon alan 56 yaşındaki olgumuzda ise takipte bulguların gerilediği gözlendi.

Pediatrik hasta grubunda; sinyal değişiklikleri muhtemel bu düzeydeki yüksek tiamin bağımlı metabolizma nedeniyle sıklıkla bazal ganglionlar düzeyinde ve karakteristik olarak putamende izlenmektedir. Buna paralel olarak tek pediatrik hastamız olan 15 yaşında olguda da putamen tutulumu tespit edildi. Ayrıca 55 yaşında Guillain-Barre nedeniyle takipli ve malnütrisyon nedeniyle WE tanısı konan olgumuzda da putamen tutulumu mevcut idi. Bununla birlikte, MRG'de sinyal değişikliklerinin tespit edilmemesi, WE tanısını dışlamamaktadır. Kontrast madde uygulanması sonrası bu olgularda T1 ağırıklı görüntülerde kontrast tutulumu gözlenebilmektedir (20). Bu bulgu sıklıkla alkoliklerde ve mamiller cisimde; hatta bazen de hastalığın tek bulgusu olarak ortaya çıkabilir $(7,21)$. Zuccolli ve ark. $(7,14)$ yayımladıkları 2 çalışmada da en sık kontrast tutulumunu mamiller cisimde bildirmiştir. Bu çalışmalarda T2 ağırlıklı görüntülerde en sık tutulum bölgesi olan talamus medialinde, mamiller cisme kıyasla daha düşük oranda (\%50) kontrast tutulumu gözlenmiştir.

Difüzyon ağırlıklı görüntülemede hastalığın patolojik mekanizması ile korele olarak; vazojenik ödem ile uyumlu apparent diffusion coefficient (ADC) serilerinde normal/artmış değerler veya sitotoksik ödem ile uyumlu düşük değerler (difüzyon kısıtılığı) tespit edilebilir. Difüzyon kısıtlılığı bulguları tedavi ile geri dönebilir. Ayrıca olguların bir kısmında difüzyon ağırlıklı görüntülemede sadece bilateral talamusta ve periakuaduktal bölgede hafif artmış sinyal şeklinde izlenebilir. Literatürde difüzyon ağırlıklı görüntülemenin 
rolü veya katkısı net olarak ortaya konulmamıştır $(15,16)$. Hasta grubumuzda difüzyon kısıtılığı tespit edilen olguların yarısında (4/8) bulgular geri dönerken, tanı konulmasını takiben kısa sürede kaybedilen 4 hastanın tümünde difüzyon kısıtlanması gösteren lezyon varlığı saptanması dikkat çekiciydi.

Alkolik ve non-alkolik hastaların kraniyal MR görüntüleri akut evrede farklılıklar gösterebilmektedir. Literatürde bu konuda 56 hasta ile en geniş seriye sahip Zuccoli ve ark. (14) 2009'da yayımladıkları çalışmalarında, atipik sinyal değişikliklerinin sadece non-alkolik grupta tespit edildiğini, tipik görüntüleme bulgularına ise daha çok alkoliklerde rastlandığını bildirmiştir. Buna neden olarak da patofizyolojik süreç farklılığı veya alkolün atipik lezyon oluşan alanlarda olası koruyucu etkisini öne sürmüştür. Ancak daha sonra başta Ha ve ark. (22), olmak üzere tipik ve atipik sinyal değişikliklerine her iki grupta da rastlandığını bildiren çok sayıda yayın ve olgu bildirimi yapılmıştır (23). Tamamı non-alkolik bireyler olan kendi hasta grubumuzda bu yayınlara paralel olarak ağırlıklı tipik görüntüleme bulguları mevcut olup, 3 hastamızda atipik tutulum bulgularından hiçbiri gözlenmedi. Bunun yanı sıra yine alkoliklerde mamiller cisim ve serebellar vermiste akut dönemde atrofi varlığı da gözlenebilmektedir. Bu durumun tekrarlayan, eski ataklara bağlı olduğu düşünülmektedir $(10,15)$. Hasta grubumuzda tanımlanan bölgelerde atrofi tespit edilmedi.

WE tanısı netleşen veya şüpheli olgularda zaman geçirmeden intravenöz tiamin replasmanı yapılmalıdır. Erken tedavi hastalığın klinik seyri açısından son derece önemlidir. Tedavi edilmeyen veya tedavisi geciken hastaların \%85'inde Korsakoff sendromu gelişebilir (sıklıkla alkoliklerde) ve \%20'sinde ölüme neden olabilir (24). Tedaviye yanıt ilk haftada gözlenebilmekle birlikte bulguların gerilemesi 1 yıla yakın bir süreyi bulabilir $(10,15)$. Bununla birlikte, nistagmus ve ataksi gibi sekel bozukluklar sıklıkla gözlenebilir. Bu bulgulara paralel olarak çalışma grubumuzdaki 6 hastamızda (3'ünde kısmi) klinik bulgular geriledi.

Retrospektif bir değerlendirme olması, hasta sayımızın azlığı, MR görüntülemede kontrast madde verilmemesi, hastaların hepsinin tedavi sonrası MR görüntülemelerinin bulunmaması çalışmamızın kısıtılıkları arasında sayılabilir.

\section{Sonuç}

Büyük oranda nonspesifik klinik bulgular göstermesi nedeniyle sıklıkla atlanabilen WE tanısında karakteristik görüntüleme bulguları ile kraniyal MR çok değerli bir yöntemdir. Tamamı non-alkolik WE tanılı olgulardan oluşan hastalarımızın görüntüleme bulguları, alkolik WE hastalarında gözlenen görüntüleme bulguları ile belirgin farklılık göstermemekte olup WE düşünülen hastaların tanı ve tedavilerinin gecikmemesi amacıyla MRG kullanımının önemli rol oynadığı sonucuna varılmıştır.

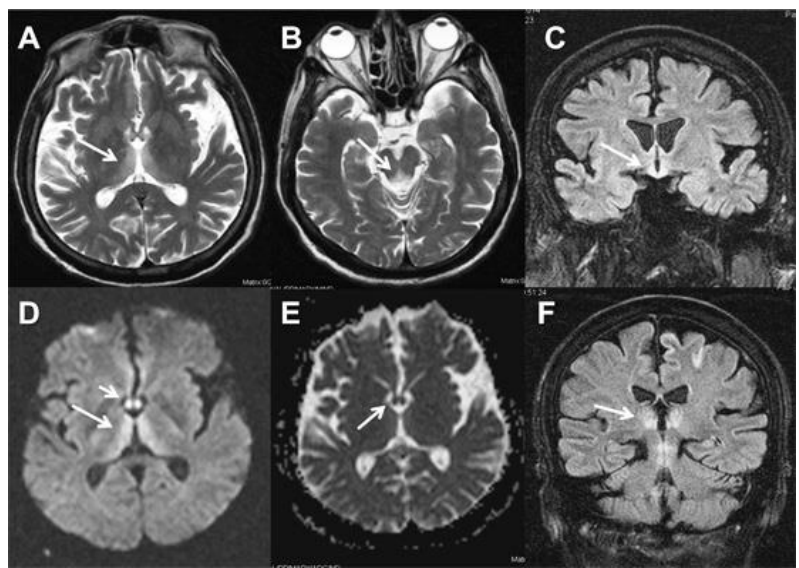

Şekil-1. Altmış beş yaşında bilinç bulanıklığı ile başvuran Crohn hastalığı tanılı olgu. Tipik WE görüntüleme bulguları ile bassvuran hastada tiamin replasmanı sonrası tam iyileşme izlendi. T2 aksiyel görüntülerde $(A, B)$ bilateral medial talamik lokalizasyonda (A-ok) ve periakuaduktal bölgede (B-ok), koronal FLAIR görüntüde (C) ise bilateral mamiller cisimlerde (C-ok) sinyal intensite artımı izlenmektedir. Difüzyon (B1000) (D) ve ADC haritalarda (E) bilateral talamus (D-ok) ve mamiller cisimlerde (D-kısa ok) difüzyon kısıtlanmas ile koronal FLAIR görüntüde (F) bilateral talamik sinyal intensite artışı (F-ok) mevcuttur.

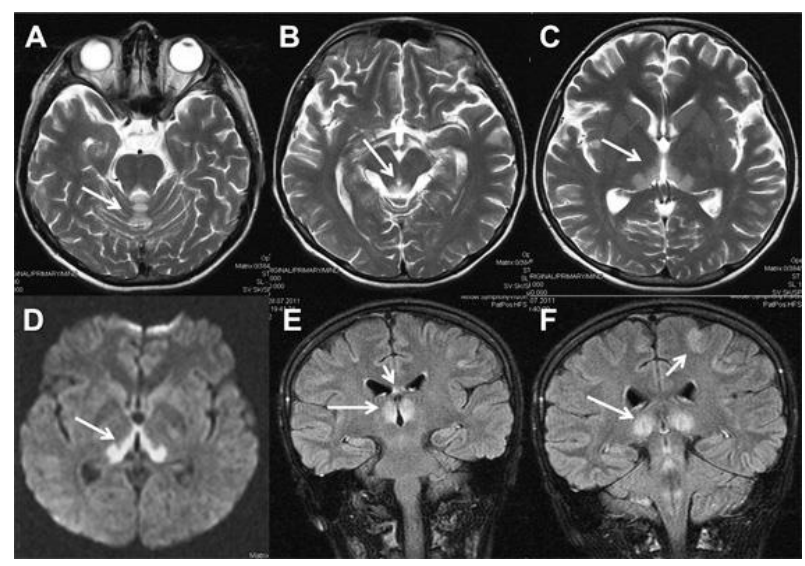

Şekil-2. Nöbet ve bilinç bulanıklığı gelişen kronik pankreatit ve buna bağlı beslenme bozukluğu olan 15 yașındaki olgu. Tipik ve atipik WE görüntüleme bulgularının birlikte izlendiği hasta uygun tedaviye rağmen kaybedildi. T2 aksiyel görüntülerde (A-C) serebellumda (A-ok), periakuaduktal alanda (B-ok), bilateral talamik lokalizasyonda (C-ok) sinyal intensite artımı izlenmektedir. Difüzyon (B1000) görüntüde bilateral medial talamik (D-ok) lokalizasyonda difüzyonel kısıtlanma mevcut. Koronal FLAIR görüntülerde (E-F) bilateral talamik lokalizasyonda (E-uzun ok, F-uzun ok), bilateral fornikste (E-kısa ok) ve sol pariyetal kortekste (F-kısa ok) sinyal intensite artışı izlenmektedir. 


\section{Kaynaklar}

1. Saad L, Silva LF, Banzato CE, Dantas C, Garcia C. Anorexia nervosa and Wernicke-Korsakoff syndrome: A case report. J Med Case Rep 2010;4:217.

2. Chiossi G, Neri I, Cavazzuti M, Basso G, Facchinetti F. Hyperemesis gravidarum complicated by Wernicke encephalopathy: background, case report, and review of the literature. Obstet Gynecol Surv 2006;61(4):255-68.

3. Loh Y, Watson WD, Verma A, Chang ST, Stocker DJ, Labutta RJ. Acute Wernicke's encephalopathy following bariatric surgery: Clinical course and MRI correlation. Obes Surg 2004;14(1):129-32.

4. Sequeira Lopes da Silva JT, Almaraz Velarde R, Olgado Ferrero F, et al. Wernicke's encephalopathy induced by total parental nutrition. Nutr Hosp 2010;25(6):1034-6.

5. Azim W, Walker R. Wernicke's encephalopathy: A frequently missed problem. Hosp Med 2003;64(6):326-7.

6. Harper CG, Giles M, Finlay-Jones R. Clinical signs in the Wernicke-Korsakoff complex: A retrospective analysis of 131 cases diagnosed at necropsy. J Neurol. Neurosurg Psychiatry 1986;49(4):341-45.

7. Zuccoli G, Gallucci M, Capellades J, et al. Wernicke encephalopathy: MR findings at clinical presentation in twenty-six alcoholic and nonalcoholic patients. AJNR Am J Neuororadiol 2007;28(7):1328-31.

8. Harper C. The incidence of Wernicke's encephalopathy in Australia-a neuropathological study of 131 cases. J Neurol Neurosurg Psychiatry 1983;46(7):593-8.

9. Harper C, Butterworth R. Nutritional and metabolic disorders. In: Graham DI, Lantos PL (eds). Greenfield's Neuropathology. Vol. 1, $6^{\text {th }}$ ed. London: Hodder Arnold;1997:601-52.

10. Zhong C, Jin L, Fei G. MR Imaging of nonalcoholic Wernicke encephalopathy: A follow-up study. AJNR Am J Neuroradiol $2005 ; 26(9): 2301-5$

11. Thomson AD. Mechanisms of vitamin deficiency in chronicalcohol misusers and the development of the Wernicke Korsakoff syndrome. Alcohol Alcohol Suppl 2000;35(1):2-7.

12. Park SH, Kim M, Na DL, Jeon BS. Magnetic resonance reflects the pathological evolution of Wernicke encephalopathy. $J$ Neuroimaging 2001;11(4):406-11.

13. Gui QP, Zhao WQ, Wang LN. Wernicke's encephalopathy in nonalcoholic patients: Clincal and pathologic features of three cases and literature reviewed. Neuropathology 2006;26(3):231-5.

14. Zuccoli G, Santa Cruz D, Bertolini M, et al. MR imaging findings in 56 patients with Wernicke encephalopathy: nonalcoholics may differ from alcoholics. AJNR Am J Neuororadiol. 2009;30(1):171-6.

15. Manzo G, De Gennaro A, Cozzolino A, Serino A, Fenza G, Manto A. MR imaging findings in alcoholic and nonalcoholic acute Wernicke's encephalopathy: A review. Biomed Res Int 2014;2014(4):503-96.

16. Zuccoli G, Pipitone N. Neuroimaging findings in acute Wernicke's encephalopathy: Review of the literature. AJR Am J Roentgenol 2009;192(2):501-8.

17. Victor M, Adams RD, Collins GH. The Wernicke-Korsakoff syndrome and related neurological disorders due to alcoholism and malnutrition. 2nd ed. Philadelphia: FA Davis Company; 1989:123-9

18. Bae SJ, Lee HK, Lee JH, Choi CG, Suh DC. Wernicke's encephalopathy: Atypical manifestation at MR imaging. AJNR Am J Neuroradiol 2001;22(8):1480-2.

19. Cui HW, Zhang BA, Peng T, Liu Y, Liu YR. Wernicke's in a patient with acute pancreatitis: Unusual cortical involvement and marvelous prognosis. Neurol Sci 2012;33(3):615-8.

20. Mascalchi M, Simonelli P, Tessa C, et al. Do acute lesions of Wernicke's encephalopathy show contrast enhancement? Report of three cases and review of the literature. Neuroradiology 1999;41(4):249-54.

21. Shogry ME, Curnes JT. Mamillary body enhancement on MR as the only sign of acute Wernicke encephalopathy. AJNR Am $J$ Neuroradiol 1994;15(1):172-4.

22. Ha ND, Weon YC, Jang JC, Kang BS, Choi SH. Spectrum of MR imaging findings in Wernicke encephalopathy: Are atypical areas of involvement only present in nonalcoholic patients? AJNR Am J Neuroradiol 2012;33(7):1398-402.

23. Sugai A, Kikugawa K. Atypical MRI findings of Wernicke encephalopathy in alcoholic patients. AJR Am J Roentgenol 2010;195(5):W372-3

24. Sechi G, Serra A. Wernicke's encephalopathy: New clinical settings and recent advances in diagnosis and management. Lancet Neurol 2007;6(5):442-55. 